\title{
Padded Headgear does not Reduce the Incidence of Match Concussions in Professional Men's Rugby Union: A Case-control Study of 417 Cases
}

\author{
Authors \\ Keith A. Stokes ${ }^{1,2}$ (D, Matthew Cross ${ }^{1,3}$, Sean Williams ${ }^{1}$ D, Carly McKay ${ }^{1}$, Brent E. Hagel4, 5, 6, 7, Stephen W. West ${ }^{1}$ D, \\ Simon Paul Roberts ${ }^{1}$, Ricardo T. Sant'Anna ${ }^{1}$, Edward Morrison ${ }^{1}$, Simon Kemp ${ }^{2,8}$
}

Affiliations

1 Department for Health, University of Bath, Bath, United Kingdom of Great Britain and Northern Ireland

2 Medical Services, Rugby Football Union, Twickenham, United Kingdom of Great Britain and Northern Ireland

3 Rugby Department, Premiership Rugby, Twickenham, United Kingdom of Great Britain and Northern Ireland

4 Sport Injury Prevention Research Centre, Faculty of Kinesiology, University of Calgary, Calgary, Canada

5 Department of Pediatrics, University of Calgary Cumming School of Medicine, Calgary, Canada

6 O'Brien Institute of Public Health, University of Calgary Cumming School of Medicine, Calgary, Canada

7 Alberta Children's Hospital Research Institute, University of Calgary Cumming School of Medicine, Calgary, Canada

8 Faculty of Epidemiology and Public Health, London School of Hygiene and Tropical Medicine Faculty of Epidemiology and Public Health, London, United Kingdom of Great Britain and Northern Ireland

\section{Key words}

traumatic brain injury, mTBI, sports injury, injury prevention, equipment, concussion severity

accepted $\quad 15.12 .2020$

published online $\quad 19.02 .2021$

Bibliography

Int J Sports Med 2021; 42: 930-935

DOI 10.1055/a-1345-9163

ISSN $0172-4622$

(c) 2021. Thieme. All rights reserved.

Georg Thieme Verlag KG, Rüdigerstraße 14, 70469 Stuttgart, Germany
Correspondence

Dr. Keith A. Stokes

Department for Health

University of Bath

Claverton Down,

BA2 7AY Bath

United Kingdom of Great Britain and Northern Ireland

Tel.: +44(0)1225 384190, Fax: +44(0)1225383833

k.stokes@bath.ac.uk

\section{ABSTRACT}

Concussion is the most common match injury in rugby union. Some players wear padded headgear, but whether this protects against concussion is unclear. In professional male rugby union players, we examined: (i) the association between the use of headgear and match concussion injury incidence, and (ii) whether wearing headgear influenced time to return to play following concussion. Using a nested case-control within a cohort study, four seasons (2013-2017) of injury data from 1117 players at the highest level of rugby union in England were included. Cases were physician-diagnosed concussion injuries. Controls were other contact injuries (excluding all head injuries). We determined headgear use by viewing video footage. Sixteen percent of cases and controls wore headgear. Headgear use had no significant effect on concussion injury incidence (adjusted odds ratio $=1.05,95 \% \mathrm{Cl}: 0.71-1.56$ ). Median number of days absent for concussion whilst wearing headgear was 8 days, compared with 7 days without headgear. Having sustained a concussion in the current or previous season increased the odds of concussion more than four-fold (odds ratio $=4.55$, $95 \% \mathrm{Cl}: 3.77-5.49)$. Wearing headgear was not associated with lower odds of concussions or a reduced number of days' absence following a concussion.

\section{Introduction}

Concussion is the most common match injury in professional men's rugby union and accounted for $21 \%$ of all injuries in the highest level of rugby in England between 2014/2015 and 2018/2019 [1]. There are a variety of strategies that could be employed to target a reduction in concussion risk in collision sports, including policy and rule changes (e.g., modifying contact [2,3]), injury prevention exercise programmes [4,5], and wearing protective equipment [6]. In relation to protective equipment, rugby union players are allowed to wear padded headgear [7]. World Rugby, the international governing body for rugby union, state that padded headgear is "not intended nor expected to protect against any form of mild traumatic brain injury" [8], but research evidence regarding a protective effect against concussion is unclear at present [9]. 
In observational studies of rugby union, there is mixed evidence as to whether there is a reduced incidence of concussion in those wearing headgear. In a sample of over 750 professional rugby players over three seasons, overall concussion incidence was 4.1/1000 hours, and there was a lower incidence of concussion in players wearing headgear $(2.0 / 1000 \mathrm{~h}, 95 \% \mathrm{Cl}: 1.0-4.1)$ than in those who did not (4.6/1000 h, $95 \% \mathrm{Cl}: 3.7-5.7)$ [10]. Supporting these findings, a cohort study of 3000 non-professional rugby players found those who self-reported always wearing headgear had a lower incidence of concussion (rate ratio $=0.57,95 \% \mathrm{Cl}: 0.40-0.82$ ) [11]. In contrast, a cohort study of 304 non-professional players over a single season showed that padded headgear tended to prevent damage to the scalp and ears (rate ratio $=0.59,95 \% \mathrm{Cl}: 0.19-1.86$ ), but that the incidence of concussion was not lessened by the use of padded headgear (rate ratio $=1.13,95 \% \mathrm{Cl}: 0.40-3.16$ ) [12].

Two intervention studies have focussed on the effect of wearing headgear on concussion incidence in rugby union. In a singleseason study involving 294 school players, there was no difference in concussion incidence in a headgear group (1179 player exposures, 7 concussions) when compared with a control group (357 player exposures, 2 concussions) [13]. Similarly, in a larger cluster randomised controlled trial involving 3686 young male players (aged 12-21 years), compared with not wearing headgear, wearing "standard" (incidence rate ratio $=1.11,95 \% \mathrm{Cl}: 0.69-1.21$ ) or "modified" (incidence rate ratio $=1.01,95 \% \mathrm{Cl}$ : 0.67-1.69) headgear did not reduce incidence of concussion when compliance was taken into account.[14] The authors did explicitly note potential for bias associated with a range of factors, highlighting poor compliance as a major limitation.

It is notable that all of the studies described above were published before 2010, and it is very likely that at this time concussion was dramatically under-reported [15]. In professional rugby union, since 2011 there has been an operational definition of concussion that has improved the detection and recording of concussion, which has contributed to an increase in concussion incidence [1]. An operational definition of concussion, routine surveillance of all injuries, and the availability of video footage for all matches offers more accurate recording of both concussion and headgear use (using video) than in previous studies. In addition, in the observational studies, previous concussion history has not been included as a confounder, despite the fact that previous concussion might influence a player's choice as to whether or not to wear headgear. The primary aim of this study was to examine the association between the use of headgear and match concussion injuries in professional male rugby union players. The secondary aim was to determine whether wearing headgear influences time to return to play following concussion.

\section{Materials and Methods}

\section{Study design and participants}

This is a nested case-control study within a larger longitudinal cohort study, and was conducted in a population of 1117 first-team male professional rugby union players (2554 player seasons) competing at the highest level of rugby in England. The study is a secondary analysis of data collected as part of the Professional Rugby
Injury Surveillance Project over four seasons (2013-2017) from all clubs participating in the English Premiership in each season. A favourable ethical opinion for all injury data collection was given by the Research Ethics Approval Committee for Health at the University of Bath and written informed consent was obtained from each player on an annual basis. The study was carried out according to the ethical standards of the journal [16]. We did not directly include patient and public involvement, but the project steering group includes key stakeholders (i.e., policy-makers, coaches, practitioners, player representatives). There was no a priori sample size calculation, but the recommendation that 200 injury cases are needed to detect small-to-moderate associations between risk factors and injury [17] was considered.

\section{Procedures}

Injury data were collected as part of the Professional Rugby Injury Surveillance Project for which club medical staff reported all match injuries via a standard electronic injury report form (Rugby Squad, The Sports Office UK Ltd). Injuries were included if they resulted in time loss, which was defined as preventing a player from fully participating in all training and match activities typically planned for that day for a period of greater than $24 \mathrm{~h}$ [18]. As part of the injury report, the match event associated with the injury was recorded and subsequently characterised as a contact (i. e., associated with tackles, scrums, rucks, mauls, lineouts, or collisions) or non-contact (i. e., associated with running or kicking) injury. Specific injury diagnoses were also recorded. Physician-diagnosed concussion was recorded according to the operational definition that was developed to support the Head Injury Assessment (HIA) process for use in elite rugby union. The HIA process has been described previously [19] and is a three-stage process that incorporates: (i) HIA01, ingame, immediate removal of players showing clear signs of concussion, or, where a meaningful head impact has occurred, an offfield screening process supported by an abridged version of the Sports Concussion Assessment Tool (SCAT); (ii) HIA02, a detailed post-game medical assessment within $3 \mathrm{~h}$ of the head impact event supported by the Sports Concussion Assessment Tool (SCAT); and (iii) HIA03, a detailed medical assessment 36-48 h after the head impact event to monitor clinical progress and to confirm or refute a diagnosis of concussion, supported by the Sports Concussion Assessment Tool (SCAT). Ultimately, experienced team doctors made a final diagnosis of concussion, supported by the HIA process, based on positive clinical signs or symptoms or clinical judgement at any of the three stages.

Each injury report recorded whether or not the player was wearing headgear at the time of the injury. To verify practitioner-reported headgear use, video footage of matches was reviewed by two members of the research team (RTS and EM) to determine whether each injured player was wearing headgear during the match. Permissible headgear is clearly defined by World Rugby [8], and as such there is no ambiguity about whether a player should or should not be included as wearing headgear. Headgear use data from the video review was used in all further analysis.

For calculation of incidence rates, exposure to match play was calculated based on 15 players per team playing for 80 min per match (i. e., $2400 \mathrm{~min}$ of exposure per match) multiplied by the total number of matches played. Injury severity was reported as 
the number of days absent from full training and/or match play as a result of an injury.

Cases were players who sustained physician-diagnosed concussion injuries. Controls were players who sustained other contact injuries (excluding all head injuries); these were chosen as controls to allow comparison of injuries with a similar mechanism as concussion (i. e., physical contact leading to injury rather than gradual onset injuries or soft-tissue injuries that did not involve contact). A supplementary analysis was conducted using all other injuries to body locations other than the head (i. e., including non-contact time-loss injuries) as the control.

\section{Data analysis}

Agreement between practitioner-reported and video verified headgear use was determined by calculating Cohen's kappa [20]. All estimations were made using $R$ (version 3.6.0; R Foundation for Statistical Computing, Vienna, Austria). A generalised linear mixed effects model was used to produce odds ratio (OR) estimates with $95 \%$ confidence intervals (Cls) for the effect of headgear use on contact injury rates. This model was implemented via the Ime4 package assuming a binomial distribution with logit link function [21]. Covariates included in the model were selected a priori based on prior knowledge and available data. The included covariates were age, positional group (forward/back), playing season, and number of concussion events in the current or previous season [22]. There were three missing data points for the positional group covariate and 101 missing data points for age. No imputation was performed for missing data as these were assumed to be 'missing completely at random' and so would not bias the model estimates. The nested case-control design allowed the number of concussion events in the current or previous season to be collated from the longitudinal cohort study data set. Confounders that we could not account for in the data included lifelong concussion history, any premorbid conditions that might affect the risk of concussion or recovery time, and risk-taking or aggressive behaviour. Player identity and team were included as random effects to account for repeated observations and clustering, and were assumed to be normally distributed (with mean 0 ). The severity distributions for concussion injuries were compared between those wearing headgear and those without headgear using the Kaplan-Meier method and log-rank test [23]. All estimates are presented with $95 \%$ confidence intervals.

\section{Results}

Incidence of concussion, and all injuries and contact injuries over each year of the study are shown in $>$ Table 1. In total, 2453 inju- ries were recorded, but video footage and injury report data could not be linked for 206 of these injuries (primarily because video footage of the match was not available). From the remaining 2247 injuries, 417 concussion cases and 1239 non-concussion contact injury controls were identified. Injury reports and video verification showed moderate agreement (92\% agreement; kappa, 0.66, 95\% $\mathrm{Cl}: 0.62-0.71)$ for headgear use in all injuries, concussion cases (94\% agreement; kappa, 0.79 , $95 \% \mathrm{Cl}: 0.71-0.87$ ), and of non-concussion contact injury controls (90\% agreement; kappa, 0.62, $95 \%$ $\mathrm{Cl}: 0.56-0.68)$. All subsequent analysis used headgear use data from the video review.

- Table 2 shows the variables by case and control status. Of the 417 concussion cases, 67 (16\%) were confirmed to be wearing headgear. Of the 1239 non-concussion contact injury controls, 199 (16\%) were confirmed to be wearing headgear. Headgear use had no significant effect on concussion injury incidence after adjusting for relevant covariates (adjusted odds ratio $=1.09$, $95 \% \mathrm{Cl}$ : $0.74-$ 1.63; Table 2). The headgear effect was consistent in the unadjusted model (odds ratio $=1.02$, $95 \% \mathrm{Cl}: 0.72-1.43$ ), when contact head injuries were included in the controls (adjusted odds ratio $=1.07,95 \% \mathrm{Cl}: 0.72-1.58$ ) and when non-contact injuries were included in the controls (adjusted odds ratio $=1.13,95 \% \mathrm{Cl}$ : $0.77-1.65)$. There was no significant difference in the severity distribution of concussion injuries incurred whilst wearing headgear versus concussions incurred without headgear (median difference $=1$ day, $P=0.63 ;$ - Fig. 1 ), with median time-loss of 8 days and 7 days, respectively. Having sustained a concussion in the current or previous season increased concussion injury incidence more than four-fold regardless of headgear status (adjusted odds ratio $=4.55$, $95 \%$ Cl: 3.77-5.49; - Table 2). Of all cases wearing headgear, $93 \%$ had sustained a concussion in the current or previous season, compared with $99 \%$ for cases not wearing head gear, $32 \%$ for controls wearing headgear, and $33 \%$ for controls not wearing headgear. A relatively low number of contact injuries (excluding concussions) in 2015-16 compared with the reference season (2013-14), coupled with a higher number of concussions ( $\vee$ Table 1 ) resulted in a significant odds ratio for the $2015-16$ season (adjusted odds ratio $=1.57,95 \% \mathrm{Cl}: 1.05-2.35$ ). Playing position (adjusted odds ratio $=0.94,95 \% \mathrm{Cl}: 0.70-1.25)$ and age (adjusted odds ratio $=0.98$, $95 \% \mathrm{Cl}: 0.94-1.02)$ were not significantly associated with concussion injury incidence.

\section{Discussion}

We examined the use of headgear and concussion injury in professional rugby union players. The main finding was that, in a setting

- Table 1 Match exposure, and number and incidence of all injuries, concussions and all other contact injuries (excluding concussions). Mean (95\% Cls) are reported per $1000 \mathrm{~h}(/ 1000 \mathrm{~h})$.

\begin{tabular}{|l|l|l|l|l|l|l|l|}
\hline \multirow{2}{*}{ Year } & \multirow{2}{*}{$\begin{array}{l}\text { Match } \\
\text { exposure (hours) }\end{array}$} & \multicolumn{2}{|c|}{ All injuries } & \multicolumn{2}{c|}{ Concussion } & \multicolumn{2}{c|}{ Non-concussion contact injuries } \\
\cline { 3 - 8 } & $\mathbf{N}=$ & Incidence $(/ \mathbf{1 0 0 0} \mathbf{h})$ & $\mathbf{N}=$ & Incidence $(/ \mathbf{1 0 0 0} \mathbf{h})$ & $\mathbf{N}=$ & Incidence $(/ \mathbf{1 0 0 0} \mathbf{h})$ \\
\hline $2013-14$ & 8160 & 645 & $79.0(73.2-85.4)$ & 80 & $9.8(7.9-12.2)$ & 399 & $48.9(44.3-53.9)$ \\
\hline $2014-15$ & 8200 & 577 & $70.4(64.9-76.3)$ & 93 & $11.3(9.3-13.9)$ & 316 & $38.5(34.5-43.0)$ \\
\hline $2015-16$ & 7162 & 446 & $62.3(56.8-68.3)$ & 113 & $15.8(13.1-19.0)$ & 241 & $33.6(29.7-38.2)$ \\
\hline $2016-17$ & 8100 & 785 & $96.9(90.4-103.9)$ & 170 & $21.0(18.1-24.4)$ & 427 & $52.7(47.9-58.0)$ \\
\hline All Years & $\mathbf{3 1 6 2 2}$ & $\mathbf{2 4 5 3}$ & $\mathbf{7 7 . 6}(\mathbf{7 4 . 6 - 8 0 . 7})$ & $\mathbf{4 5 6}$ & $\mathbf{1 4 . 4 ( 1 3 . 2 - 1 5 . 8 )}$ & $\mathbf{1 3 8 3}$ & $\mathbf{4 3 . 7 ( 4 1 . 5 - 4 6 . 1 )}$ \\
\hline
\end{tabular}


- Table 2 Distribution of variables and results of the logistic regression analysis for the concussion cases and contact-injury controls.

\begin{tabular}{|c|c|c|c|c|}
\hline Predictor Variable & Cases $(n=417)$ & Controls $(n=1239)$ & Adjusted Odds Ratio $(95 \% \mathrm{Cl})$ & $P$-value \\
\hline \multicolumn{5}{|l|}{ Headgear use } \\
\hline No & $84 \%(n=350)$ & $84 \%(n=1041)$ & reference & \\
\hline Yes & $16 \%(n=67)$ & $16 \%(n=198)$ & $1.09(0.77-1.63)$ & 0.66 \\
\hline \multicolumn{5}{|l|}{ Season } \\
\hline $2013-14$ & $19 \%(n=79)$ & $31 \%(n=384)$ & reference & \\
\hline $2014-15$ & $22 \%(n=92)$ & $24 \%(n=297)$ & $1.17(0.78-1.76)$ & 0.44 \\
\hline $2015-16$ & $26 \%(n=108)$ & $18 \%(n=223)$ & $1.57(1.05-2.35)$ & 0.03 \\
\hline $2016-17$ & $33 \%(n=138)$ & $28 \%(n=335)$ & $0.93(0.62-1.38)$ & 0.71 \\
\hline Mean \pm SD number of concussions in current/previous season & $1.5 \pm 0.9$ & $0.4 \pm 0.7$ & $4.55(3.77-5.49)$ & $<0.001$ \\
\hline No headgear & $1.5 \pm 0.9$ & $0.4 \pm 0.8$ & & \\
\hline Headgear & $1.6 \pm 1.1$ & $0.4 \pm 0.7$ & & \\
\hline Median (IQR) number of concussions in current/previous season & $1(0-2)$ & $0(0-1)$ & & \\
\hline \multicolumn{5}{|l|}{ Positional group } \\
\hline Backs & $42 \%(n=175)$ & $39 \%(n=483)$ & reference & \\
\hline Forwards & $58 \%(n=242)$ & $61 \%(n=756)$ & $0.94(0.70-1.25)$ & 0.67 \\
\hline Age (years) & $26.2 \pm 3.7$ & $26.3 \pm 3.8$ & $0.98(0.94-1.02)$ & 0.26 \\
\hline
\end{tabular}

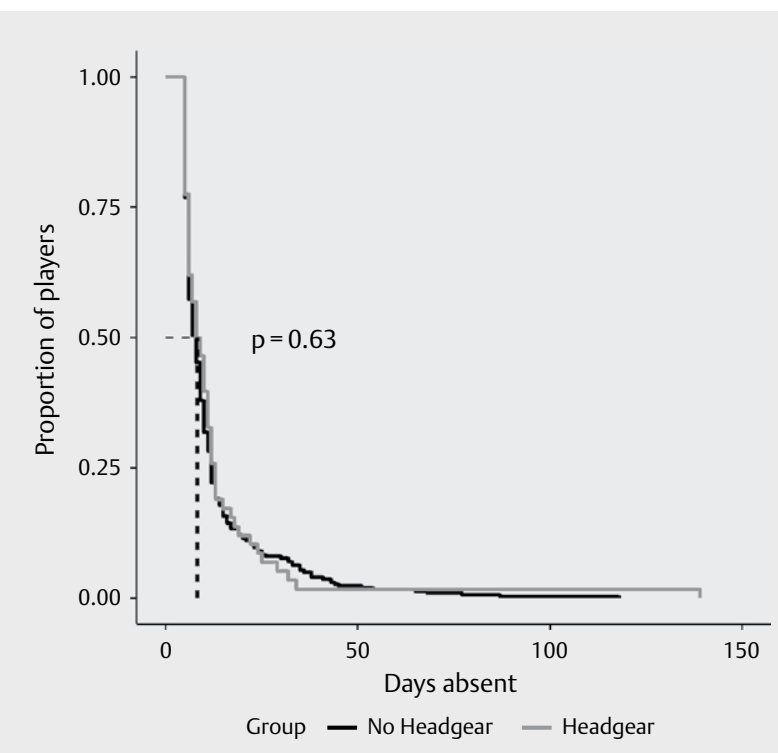

Fig. 1 Kaplan-Meier survival curve for time to return to full training or match play in concussed players wearing headgear and not wearing headgear at the time of injury.

where concussion identification and diagnosis was of a high standard and where video footage was used to verify headgear use, wearing headgear was not associated with concussion. Days' absence due to concussion was not different when players were or were not wearing headgear. The number of concussions sustained in the current or previous season was associated with an increased the odds of concussion.

\section{Why doesn't headgear reduce concussion?}

The finding that the use of headgear that is permitted by the international governing body was not associated with concussion is in agreement with intervention studies in rugby union $[13,14]$. Fur- thermore, there was no difference between wearing and not wearing headgear in terms of the number of days missed following concussion, which supports previous findings of similar acute concussion outcomes in helmeted and unhelmeted sports [24]. The primary purpose of padded headgear is to prevent abrasions and lacerations, with evidence of reduced damage to the scalp and ears when wearing headgear [12]. This could not be explored in the present study because most such injuries do not typically result in time loss; only one 24-h time loss injury abrasion or laceration to the scalp was recorded in the current data set. Headgear might also attenuate linear forces when the head does not move, but angular acceleration is an important contributor to the risk of sustaining a concussion [25], and there is no evidence that headgear can reduce head movement during impacts. As such, currently available rugby union headgear is unlikely to confer a biomechanical benefit that reduces either the incidence or severity of concussion. In this context, it is interesting to note that only $16 \%$ of players who sustained an injury were wearing headgear, reflecting relatively low levels of use.

\section{Recent concussion is a strong risk factor for concussion}

Consistent with findings other settings [26-28], previous concussion was found to be a risk factor for subsequent concussion. This might be because some individuals are at greater risk of concussion in the first place, because an initial concussion leaves an individual more vulnerable to subsequent concussion or most likely both. In fact, previous concussion has been identified as a risk factor for all injuries in a variety of sports settings [22, 29, 30], with prolonged motor system and attentional deficits highlighted as possible explanations [31]. Further work is needed to understand patterns of multiple concussions in athletes over the course of their careers to improve concussion management.

\section{Strengths and limitations}

One strength of this study is that we employed video verification to determine whether a player who sustained an injury was wearing headgear. This overcomes the challenges of using self-report 
methods or relying on practitioners to record headgear use. However, there was moderate agreement between practitioner-reported headgear use and video verified headgear use. For an injury report form field that might be considered irrelevant when reporting anything other than head injuries, this indicates good reporting compliance. In this setting, practitioner reported headgear use appears acceptable without the further need for video verification. Concussions were physician-diagnosed according to standard and rigorous criteria. In addition, given that they were also injured, we would argue controls were representative of the headgear experience of the source population that produced the concussion cases. Known confounders were addressed in the design (controls also experienced an injury event) and through regression modelling (e. g., concussion history).

Limitations to the study included that it was not possible to identify all players who sustained injuries on video, though this accounted for less than $10 \%$ of injuries and the sample size was still large. Secondly, we did not have a record of each player's entire concussion history, and therefore we could only adjust for recent concussions. It is notable, however, that a very large proportion of cases (both those wearing and not wearing headgear) had a history of concussion in the current or previous season and that the number of recent concussions was not different between players choosing to wear headgear and those choosing not to wear headgear. A further limitation is that, over the four seasons of data, the concussion incidence rate increased, indicating improved identification of concussion over this period. Although it is likely that some concussions were not identified in the earlier seasons of the study, the difference in number of concussions in each season was adjusted for in the model. It is also possible that player reporting of concussion was different in those wearing headgear and those not wearing headgear based on their previous history of and attitudes towards concussion. We could not account for this in our analysis, but the operational standards in relation to the identification of concussion in this professional league should limit any impact in this regard. We acknowledge that there are confounders that we could not account for in the data, such as premorbid conditions that might affect the risk of concussion or recovery time as well as differential management of players returning from concussion. Given that both cases and controls sustained contact injuries, confounding by risktaking or aggressive behaviour in individuals would be similar in both groups. We also acknowledge that individual exposure data was not collected in this study.

\section{Conclusion}

Professional rugby union players who wore headgear did not have lower odds of sustaining a concussion, nor did wearing headgear result in a difference in the number of days' absence following a concussion compared with not wearing headgear. This study does not provide evidence that currently available padded headgear should be recommended for preventing concussions.

\section{Funding}

This work was funded by the Rugby Football Union and Premiership Rugby.

\section{Acknowledgements}

We would like to thank staff and players at all of the Premiership Clubs for their contribution to the study.

\section{Conflicts of Interest}

KAS and STPK are employees of the Rugby Football Union, which is the governing body for rugby union in England. MJC is employed by Premiership Rugby, the body responsible for the highest-level rugby union competition in England.

\section{References}

[1] West SW, Starling L, Kemp S et al. Trends in match injury risk in professional male rugby union: A 16-season review of 10851 match injuries in the English Premiership (2002-2019): The Professional Rugby Injury Surveillance Project. Br J Sports Med 2020. Epub ahead of print. doi:10.1136/ bjsports-2020-102529

[2] Stokes KA, Locke D, Roberts S et al. Does reducing the height of the tackle through law change in elite men's rugby union (The Championship, England) reduce the incidence of concussion? A controlled study in 126 games. Br J Sports Med 2021; 55: 220-225. doi:10.1136/ bjsports-2019-101557

[3] Emery C, Palacios-Derflingher L, Black AM et al. Does disallowing body checking in non-elite 13- to 14-year-old ice hockey leagues reduce rates of injury and concussion? A cohort study in two Canadian provinces. Br J Sports Med 2020; 54: 414-420. doi:10.1136/ bjsports-2019-101092

[4] Hislop MD, Stokes KA, Williams S et al. Reducing musculoskeletal injury and concussion risk in schoolboy rugby players with a pre-activity movement control exercise programme: a cluster randomised controlled trial. Br J Sports Med 2017; 51: 1140-1146. doi:10.1136/bjsports-2016-097434

[5] Attwood M], Roberts SP, Trewartha G et al. Efficacy of a movement control injury prevention programme in adult men's community rugby union: a cluster randomised controlled trial. Br J Sports Med 2018; 52 : 368-374 . doi:10.1136/bjsports-2017-098005

[6] Chisholm DA, Black AM, Palacios-Derflingher $L$ et al. Mouthguard use in youth ice hockey and the risk of concussion: Nested case-control study of 315 cases. Br J Sports Med 2020; 54: 866-870

[7] World Rugby. Regulation 12: Provisions relating to players' dress. 2019 available at https://www.world.rugby/handbook/regulations/reg-12

[8] World Rugby. Headgear Performance Specification. 2019

[9] Emery CA, Black AM, Kolstad A et al. What strategies can be used to effectively reduce the risk of concussion in sport? A systematic review. Br J Sports Med 2017; 51: 978-984. doi:10.1136/bjsports-2016-097452

[10] Kemp SPT, Hudson Z, Brooks JHM et al. The epidemiology of head injuries in English professional rugby union. Clin J Sport Med 2008; 18: 227-234. doi:10.1097/JSM.0b013e31816a1c9a

[11] Hollis SJ, Stevenson MR, McIntosh AS et al. Incidence, risk, and protective factors of mild traumatic brain injury in a cohort of Australian nonprofessional male rugby players. Am J Sports Med 2009; 37: 2328-2333. doi:10.1177/0363546509341032

[12] Marshall SW, Loomis DP, Waller AE et al. Evaluation of protective equipment for prevention of injuries in rugby union. Int J Epidemiol 2005; 34: $113-118$

[13] McIntosh AS, McCrory P. Effectiveness of headgear in a pilot study of under 15 rugby union football. Br J Sports Med 2001; 35: 167-169. doi:10.1136/bjsm.35.3.167 
[14] McIntosh AS, McCrory P, Finch CF et al. Does padded headgear prevent head injury in rugby union football? Med Sci Sports Exerc 2009; 41: 306-313. doi:10.1249/MSS.0b013e3181864bee

[15] Fraas MR, Coughlan GE, Hart EC et al. Concussion history and reporting rates in elite Irish rugby union players. Phys Ther Sport 2014; 15: 136-142. doi:10.1016/j.ptsp.2013.08.002

[16] Harriss D], MacSween A, Atkinson G. Ethical standards in sport and exercise science research: 2020 update. Int J Sports Med 2019; 40: 813-817. doi:10.1055/a-1015-3123

[17] Bahr R, Holme I. Risk factors for sports injuries - a methodological approach. Br J Sports Med 2003; 37: 384-392. doi:10.1136/ bjsm.37.5.384

[18] Fuller CW, Molloy MG, Bagate C et al. Consensus statement on injury definitions and data collection procedures for studies of injuries in rugby union. Br J Sports Med 2007; 41: 328-331. doi:10.1136/ bjsm.2006.033282

[19] Fuller GW, Kemp SPT, Decq P. The International Rugby Board (IRB) Pitch Side Concussion Assessment trial: a pilot test accuracy study. $\mathrm{Br}$ ] Sports Med 2015; 49: 529-535. doi:10.1136/bjsports-2014-093498

[20] McHugh ML. Interrater reliability: the kappa statistic. Biochem Med 2012; 22: 276-282

[21] Bates D, Sarkar D, Bates MD et al. The Ime4 package. R package version 2007; 2: 74

[22] Cross M, Kemp S, Smith A et al. Professional Rugby Union players have a $60 \%$ greater risk of time loss injury after concussion: A 2-season prospective study of clinical outcomes. Br J Sports Med 2016; 50: 926-931. doi:10.1136/bjsports-2015-094982

[23] Therneau T A package for survival analysis in $S$. $R$ package version 236-12 2012
[24] Zuckerman SL, Lee YM, Odom M] et al. Sports-related concussion in helmeted vs. unhelmeted athletes: Who fares worse? Int J Sports Med 2015; 36: 419-425. doi:10.1055/s-0034-1395587

[25] McIntosh AS, McCrory P. Impact energy attenuation performance of football headgear. Br J Sports Med 2000; 34: 337-341. doi:10.1136/ bjsm.34.5.337

[26] Van Pelt K, Allred D, Cameron K et al. A cohort study to identify and evaluate concussion risk factors across multiple injury settings: Findings from the CARE Consortium. Inj Epidemiol 2019; 6: 1. doi:10.1186/s40621-018-0178-3

[27] Abrahams S, Mc Fie S, Patricios J et al. Risk factors for sports concussion: An evidence-based systematic review. Br J Sports Med 2014; 48: 91-97. doi:10.1136/bjsports-2013-092734

[28] Brett BL, Kuhn AW, Yengo-Kahn AM et al. Risk factors associated with sustaining a sport-related concussion: an initial synthesis study of 12,320 student-athletes. Arch Clin Neuropsych 2018; 33: 984-992. doi:10.1093/arclin/acy006

[29] Nordstrom A, Nordstrom P, Ekstrand J. Sports-related concussion increases the risk of subsequent injury by about $50 \%$ in elite male football players. Br J Sports Med 2014; 48: 1447-1450. doi:10.1136/ bjsports-2013-093406

[30] Lynall RC, Mauntel TC, Padua DA et al. Acute lower extremity injury rates increase after concussion in college athletes. Med Sci Sports Exerc 2015; 47: 2487-2492. doi:10.1249/mss.0000000000000716

[31] Howell DR, Lynall RC, Buckley TA et al. Neuromuscular control deficits and the risk of subsequent injury after a concussion: a scoping review. Sports Med 2018; 48: 1097-1115. doi:10.1007/s40279-018-0871-y 